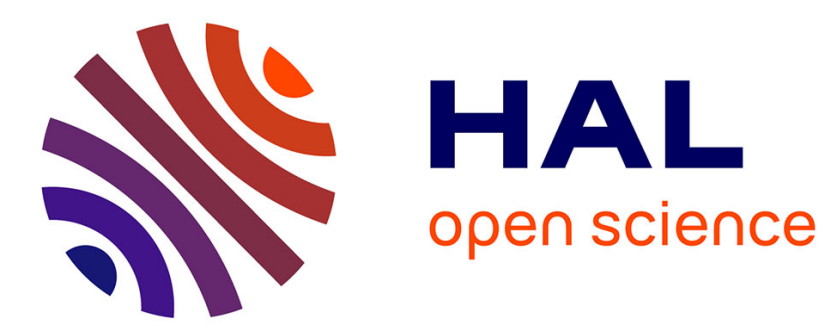

\title{
Inter-cellular scheduler for 5G wireless networks
}

Cédric Gueguen, Mahdi Ezzaouia, Mohamad Yassin

\section{To cite this version:}

Cédric Gueguen, Mahdi Ezzaouia, Mohamad Yassin. Inter-cellular scheduler for 5G wireless networks. Physical Communication, 2016, 10.1016/j.phycom.2015.10.005 . hal-01293199

\section{HAL Id: hal-01293199 \\ https://hal.science/hal-01293199}

Submitted on 12 May 2016

HAL is a multi-disciplinary open access archive for the deposit and dissemination of scientific research documents, whether they are published or not. The documents may come from teaching and research institutions in France or abroad, or from public or private research centers.
L'archive ouverte pluridisciplinaire HAL, est destinée au dépôt et à la diffusion de documents scientifiques de niveau recherche, publiés ou non, émanant des établissements d'enseignement et de recherche français ou étrangers, des laboratoires publics ou privés. 


\title{
Inter-Cellular Scheduler for 5G Wireless Networks
}

\author{
Cédric Gueguen, Mahdi Ezzaouia, Mohamad Yassin \\ University of Rennes 1, IRISA, Campus de Beaulieu, 35042 Rennes, France \\ Emails: \{cedric.gueguen, mahdi.ezzaouia, mohamad.yassin\}@irisa.fr
}

\begin{abstract}
Enhancing the Quality of Experience (QoE) in wireless networks is a crucial issue. Many acknowledged works focus on intra-cellular scheduling. They have shown that when the channel impairment is taken into consideration by the opportunistic scheduling approaches, it allows to reach higher throughputs and, for the most efficient ones, a higher fairness. However, if some of these works provide results near to optimum considering a single cell, high QoE cannot be guaranteed for scenarios where the cells are overloaded. In this article, we propose a new intercellular scheduler able to help the overloaded cells thanks to a dynamic cell bandwidth allocation. Our resource allocation technique is based on an adequate emergency parameter called Mean Cell Packet Delay Outage Ratio (MCPDOR). Performance evaluation shows that the proposed scheduler widely outperforms existing solutions in various scenarios. A variant of our solution that does not consider MCPDOR is also proposed and evaluated.
\end{abstract}

\section{Index Terms}

Wireless Networks, 5G, Scheduling, Quality of Experience, Quality of Service.

\section{INTRODUCTION}

Providing mobile multimedia transmission services with an adequate Quality of Service (QoS) is very challenging. In contrast with wired communications, wireless transmissions are subject to many channel impairments such as path loss, shadowing and multipath fading [1-4]. These phenomena severely affect the transmission capabilities and in turn the QoS experienced by applications, not only in terms of data integrity but also in terms of the supplementary delays or packet losses that appear when the effective bit rate at the physical layer is low. The past decades have witnessed intense research efforts on wireless digital communications. Among all the studied transmission techniques, Orthogonal Frequency Division Multiplexing (OFDM) [5] has clearly emerged for future broadband wireless multimedia networks (4G [6] and 5G [7] systems). It is already widely implemented in the most recent wireless systems like 802.11a/g or 802.16. The basic principle of OFDM for fighting the negative impact of multipath propagation is to divide the available channel bandwidth into several subfrequency bands, such that their width is less than the coherence bandwidth of the channel (inverse of the delay spread). The transmission of a high speed signal on a broadband frequency selective channel is then substituted by the transmission of slow speed signals on multiple subcarriers, which are immune to intersymbol interference and subject to flat fading. This subdivision of the overall bandwidth into multiple channels provides frequency diversity. This frequency diversity, along with the time and the multiuser diversity result in a very spectrally efficient system subject to an adequate scheduling.

In this context, much interest has recently been given to the design of intra-cellular scheduling algorithms that improve the performance of multiuser OFDM systems [8-11]. Opportunistic scheduling techniques take advantage of multiuser diversity by preferably allocating the resources to the active mobile(s) with the most favorable channel conditions at a given time. This technique was first explored in single carrier communications [12]. More recently, opportunistic scheduling has been exploited in multicarrier systems $[13,14]$. These schemes are derived from the Maximum Signal-to-Noise Ratio ${ }^{1}$ (MaxSNR) technique that allocates the resource at a given time to the active mobile with the greatest SNR. Dynamically adapting the modulation and coding allows then to always make the most efficient use of the radio resource and

\footnotetext{
${ }^{1}$ also known as Maximum Carrier to Interference ratio (MaxC/I).
} 
come closer to the Shannon limit. This maximizes the system capacity from an information theory point of view. However as much efficient the intra-cellular scheduler is, QoS and QoE cannot be guaranteed when the considered cell is overloaded. Consequently, 5G systems require new strategies in order to avoid cell overloading.

The 5G network should be able to serve massive number of UEs in the extreme cases. For instance, data rates of several tens of Mbit/s should be supported for tens of thousands of UEs in crowded areas, such as stadiums or open-air events [7]. Another example is providing $1 \mathrm{Gbit} / \mathrm{s}$ simultaneously to tens of UEs in the same office floor. 5G deals with a very large number of connections per square meter, and a huge traffic density. Moreover, it should be able to provide $10 \mathrm{~ms}$ end-to-end latency in general, and $1 \mathrm{~ms}$ end-to-end latency for the use cases that require extremely low latency [15]. Thus, more sophisticated techniques are required to manage the available spectrum efficiently and to satisfy these QoS requirements.

In this article, we propose an inter-cellular scheduler for efficient support of multimedia services in multi-user 5G wireless networks. Our solution is called Inter-cellular Bandwidth Fair Sharing scheduler (IBFS). It dynamically allocates the available bandwidth between the cells, taking into account their relative difficulties to ensure high QoS. The concept is to help the overloaded cell by allocating the potential useless part of bandwidth of its neighbors to it. Consequently, more subcarriers are allocated to the overloaded cell, which helps it to absorb its traffic congestion and decrease user dissatisfaction without penalizing the donor cells that keep only the optimal quantity of radio resource units to ensure QoS to their own users.

This article also deals with the metric used to select the best candidate cell to receive additional bandwidth from its neighbors. Indeed, the logical metric could be to base our approach on the global cell traffic load, but we will show that it is not the optimal metric. Indeed, cell's mobiles have no reason to use the same data rate profiles (less or more elastic) and the same applications with the same delay constraints. Intra-cellular schedulers of the cells with more elastic traffic profile or more restrictive QoS constraints have a more difficult task to ensure QoS. They could require more bandwidth than the schedulers of other cells with higher global throughput but less restrictive QoS constraints or lower peak data rate demands. Consequently, the IBFS scheduler should be based on a reliable metric, which is a crucial issue for its performance. Our second contribution is to propose an adequate metric in order to select the best cell to help. This metric, called Mean Cell Packet Delay Outage Ratio (MCPDOR), measures the cell emergency to access to more radio resources, and it allows to always select the appropriate cell to help.

The rest of this article is organized as follows. Section II describes the state-of-the-art techniques, including methods that consider the inter-cellular scheduling approaches. Section III describes the IBFS solution, and gives details about our proposed algorithm. In Section IV, we present a detailed performance evaluation of our proposed solution through a simulation study. Section $\mathrm{V}$ concludes the article and summarizes our contributions.

\section{RELATED WORK}

Rather than promoting standardized Inter-Cell Interference Coordination (ICIC) techniques, the Third Generation Partnership Project (3GPP) provides support for proactive and reactive schemes, and it allows constructors and operators to configure a wide range of non-standardized ICIC techniques [16-18]. We classify these techniques into centralized, decentralized, and hybrid schemes.

The centralized approach requires the existence of a central management entity. It collects information related to channel quality and UE throughput demands. Then, it finds the optimal resource allocation between the existing base stations, and it also performs resource allocation among UEs (scheduling). Although the centralized approach offers the optimal resource allocation, a large amount of signaling messages is generated. The decentralized approach allows each cell to determine its own resource allocation, without the need to cooperate with other cells. This approach does not generate any additional signaling overhead, and it is characterized by a low implementation complexity. However, it does not guarantee the optimal resource allocation. Hybrid approaches are proposed as a compromise between 
centralized and decentralized approaches. In these schemes, a centralized control entity collects channel quality information and UE throughput demands in order to adjust resource allocation between the network cells, while RB allocation to the active UEs is locally performed by each base station.

The frequency reuse-N model, Fractional Frequency Reuse (FFR), and Soft Frequency Reuse (SFR) techniques [19] have been widely suggested to minimize interference between adjacent cells. Traditionally, adjacent cells of a mobile network are grouped into clusters where only a portion of the available spectrum is used in each cell. Therefore, interference is reduced since frequency resources are not simultaneously used by adjacent base stations. If $g$ is the number of cells within a cluster (also called: cluster size), then $\frac{1}{g}$ of the available subcarriers are used in each cell according to frequency reuse- $g$ model. Figure 1 illustrates a mobile network where the frequency reuse- 3 model is used to manage frequency resources distribution between the different cells.

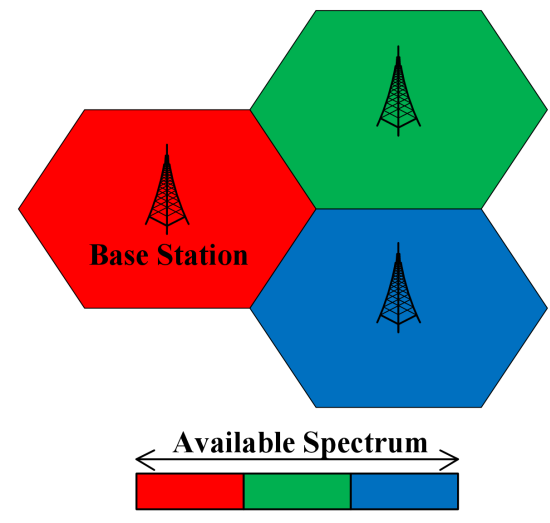

Fig. 1: Frequency reuse-3 model

FFR and SFR are not able to dynamically adapt to situations where throughput demands or UE positions are not homogeneously distributed between the different cells. To improve the performance of FFR, resource allocation and interference coordination problems are jointly considered in [20]. The proposed scheme searches for the optimal dimensions of cell-center and cell-edge zones as well as the optimal frequency reuse factor. In [21], a multi-objective algorithm for improving SFR performance is proposed. It addresses the tradeoff between enhancing network capacity and improving cell-edge performance.

A matrix-based scheduling method is proposed in [10], where the objective is to guarantee proportional fairness among the different UEs. In fact, UEs with good channel conditions and low average past throughputs are allowed to receive more resources. However, this technique does not modify resource allocation between network cells. Thus, inter-cell interference problems are not alleviated.

In this context, we introduce a heuristic resource allocation algorithm that aims at reducing InterCell Interference (ICI) and improving user satisfaction. The proposed IBFS scheduler is a hybrid ICIC approach that consists of two phases: in the first phase, bandwidth sharing between the different cells is adjusted by a central controller that manages a multi-cell cluster after receiving the necessary information from the different cells. In the second phase, resource allocation is delegated to the base stations, and UE scheduling is performed locally for each cell. It is a cooperative algorithm that dynamically adjusts resource allocation among the adjacent cells according to user satisfaction in each cell. 
TABLE I: List of Symbols

\begin{tabular}{ll}
\hline$i$ & index of the cell \\
$g_{i}$ & group of the cell $\mathrm{i}$ \\
$g$ & index of the group of cells in a cluster $(i . e . g$ is the number of different bandwidth groups in a cluster Reuse- $g$ ) \\
$g_{\max }$ & maximum number of different groups \\
$d_{g}$ & leader donor cell of group g \\
$k$ & index of the cells in a specific group \\
$r$ & cell selected to receive help from donor cell \\
$W_{g(i)}(k)$ & quantity of bandwidth, originally of the group $g$ of cell $i$, and currently owned by the cell $k$ \\
$W_{\min }$ & quantity of the bandwidth that a cell cannot lend to other cells \\
\hline
\end{tabular}

III. INTER-CELlular BANDWIDTH FAIR SHARING SCHEDUlER (IBFS)

In this Section, we describe the core of the proposed IBFS algorithm. IBFS consists in fair sharing of the bandwidth between the different cells. We propose two versions: IBF $S_{\text {load }}$ that helps the cell having the highest traffic load (defined as the total amount of data that users of the cell want to receive and transmit), and $I B F S_{M C P D O R}$ that helps the cell characterized by the highest MCPDOR. In sub-section III.A, we will describe the global IBFS mechanism, and the different parameters used across this article are defined in table I. Discussion about $M C P D O R$ and why IBF $S_{M C P D O R}$ outperforms $I B F S_{\text {load }}$ will be given in sub-section III.B.

\section{A. IBFS algorithm}

Our proposed IBFS mechanism (Fig. 2) operates as follows:

- Step 1: For each cell of the system, the inter-cellular scheduler refreshes the values of MCPDOR for $I B F S_{M C P D O R}$ and respectively the traffic load for IBF $S_{\text {load }}$. IBFS goes to the next step.

- Step 2: IBFS selects the cell that has the highest MCPDOR value for $I B F S_{M C P D O R}$, with the highest traffic load for $I B F S_{\text {load }}$. This cell is denoted $r . r$ will be the cell to help in this scheduling occurrence. All the cells in its neighborhood are potential donor cells. IBFS goes to the next step.

- Substep 3: Among all the $k$ cells in the neighborhood of $r$ and for each group of band $g$ different than the group of $r$, IBFS determines the leader donor cell $d_{g}$ that has the highest portion $W_{g(r)}(k)$ of the band $g_{r}$. This step allows to detect and select a donor cell that could have received previously the highest part of bandwidth from $r$ in order to restitute it. If $W_{g, k}$ is not null, IBFS goes to step 3.1. Otherwise, this group has no leader donor cell and the inter-cell scheduler goes to step 3.2.

- Substep 3.1: For each leader donor's group, IBFS restitutes from $d_{g}$ a total amount of frequency equal to $Q_{\text {transfer }}$ to $r$. This restitution is also made from $d_{g}$ to the cells of the same groups of $r$ neighboring $d_{g}$. This avoids future potential frequency blocking. In addition, if the cells in the neighborhood of $r$ and of the same group $d_{g}$ used frequency included in this restitution, IBFS also restitute them following the same rules. IBFS goes to step 4.

- Substep 3.2: If IBFS reaches this substep, this is because IBFS tries to help $r$ not thanks to bandwidth restitution, but taking the own bandwidth of donor cells (frequency of their band $\left.W_{g(k)}(k)\right)$ for the frequency transfer. This requires some additionnal verification. Even in the case of a cell that has no users in its coverage zone, we consider that this cell must never lend the totality of its bandwidth to its neighbors otherwise the cell disapears. We define a minimum bandwidth $W_{\min }$ which represents the part of the bandwidth that a cell must never lend to the other cells in order to always be able to offer a minimal service. Thanks to $W_{\text {min }}$, if a new user appears, he will always have minimal radio resource units available to get connected (if $W_{\min }$ is not enough, IBFS will adequalty share the available bandwidth between the cells in the 


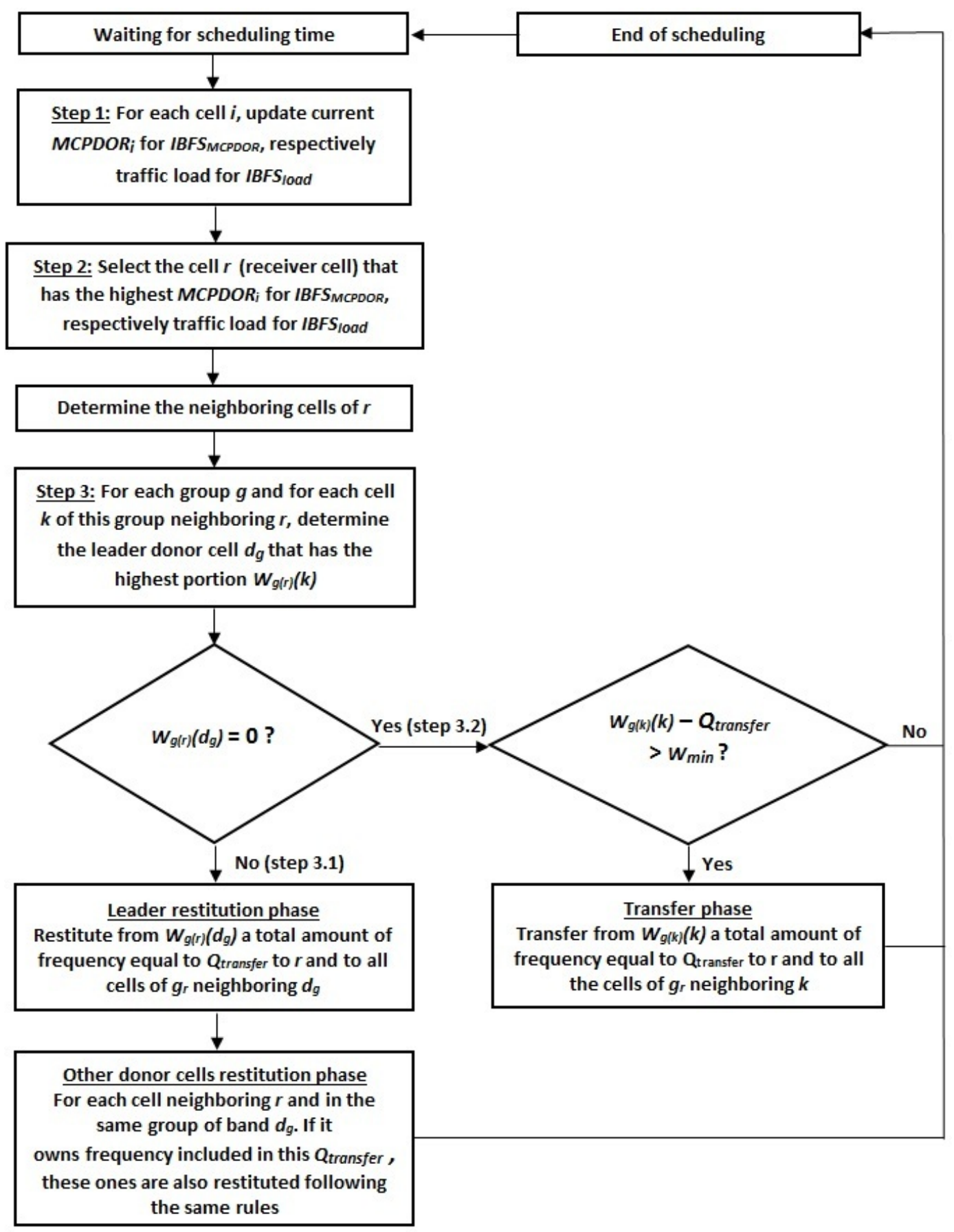

Fig. 2: IBFS scheduling algorithm flow chart.

next occurrence, and it will allocate more frequency resources to this cell). Consequently, for each group of band $g$ and for each potential donor cell $k$ of the considered group, IBFS checks whether the total bandwidth $W_{g(k)}(k)$ minus $Q_{\text {transfer }}$ is greater than or equal to $W_{\text {min }}$. If this condition is respected ${ }^{2}$, IBFS transfers $Q_{\text {transfer }}$ from all the donor cells to the cell $r$ and their respective neighbors included in the band $g_{r}$. Otherwise, helping $r$ from this group of band is impossible, and the scheduling process ends for this group without any transfer. IBFS goes to step 4.

- Step 4: End of the scheduling process.

\footnotetext{
${ }^{2}$ Note that in order to avoid interference, a cell can only use frequency given by its neighbors in the case where this cell has received this one from all its neighbors that are included in the band that originally contains this frequency.
} 


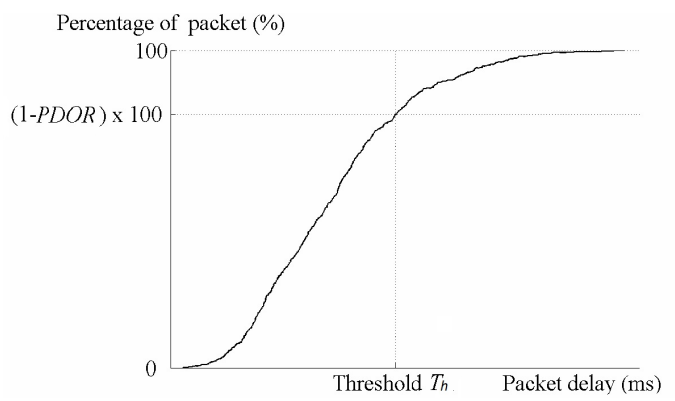

Fig. 3: Example packet delay CDF and experienced PDOR.

In order to conclude this section, we would detail one point that merit more explanation. IBFS helps cells that experience difficulties by taking frequency resources from its neighborhood. As we described in step 3, restitution (step 3.1) must be always favored when possible. Indeed, it is less costly for the system compared to the simple transfer (step 3.2). For example, if $g_{\max }=3$, giving $Q_{\text {transfer }}$ to help a cell means taking $Q_{\text {transfer }}$ from three cells in the neighborhood of $r$, while restitution means taking $Q_{\text {transfer }}$ from the leader donor and less than or equal to $Q_{\text {transfer }}$ from the two other concerned cells.

\section{B. Cell selection metric}

Considering that the cell to help is always the cell with the highest traffic load ( $I B F S_{\text {load }}$ solution) is not always optimal. Indeed, the crucial objective of IBFS is to fully support $5 \mathrm{G}$ multimedia transmission services, including the widest range of services [22]: VoIP, videoconference, email, file transfer... This requires the coexistence of delay sensitive flows with tight delay constraints and elastic traffic pattern as well as non real time traffic with looser delay constraints. For the intracellular scheduler, the most difficult flows to manage are unquestionably the delay sensitive flows. Since cells of the 5G system will manage users with different services, it appears that the cell having the highest traffic load is not the one that experiences the worst Quality of Experience (QoE). Another cell with less traffic load can have users with more delay sensitive and more elastic flows. In this case, the $I B F S_{\text {load }}$ solution will not help the cell in need.

The best solution is to select the cell that has the highest difficulties to satisfy its users. However measuring user satisfaction is a very difficult task in modern networks. The used applications are more and more varied, and require different management techniques with different QoS constraints such as jitter, mean delay, or guaranteed data rate. Considering the diversity of all the applied applications, measuring if the application is satisfied or not is really challenging. In this article, an additional contribution is to propose a new metric (the Mean Cell Packet Delay Outage Ratio $M C P D O R$ ) to measure cells users satisfaction in a generic approach, which takes into account all the potential QoS constraints to be satisfied in a simple step. $M C P D O R$ does not provide feedbacks about the nature of the problem that the applications can meet in the cell, but it alerts whether the applications of the cell users have their QoS constraints satisfied in the network or not. IBF $S_{M C P D O R}$ protocol selects the cell to help thanks to MCPDOR. This will avoid making mistakes in the cell selection, and it allows to always help the one that has the most of difficulties.

MCPDOR of the cell $i$ is the average of the Packet Delay Outage Ratio (PDOR) of all the users' service flows in this cell:

$$
M C P D O R_{i}=\frac{\sum P D O R}{\text { Total number of user's service flows in the cell }} .
$$

We define a service flow as a traffic stream and its QoS profile, in a given transmission direction. A mobile may have multiple service flows both in the uplink and in the downlink. An application may also use several service flows enabling for instance the implementation of Unequal Error Protection schemes 
in the physical layer. Each service flow possesses its own transmission buffer. The QoS profile is defined as the set of parameters that characterizes the QoS requirements of a service flow mainly in terms of data integrity and delay. In the following, data integrity requirements are specified by a Bit Error Rate (BER) target, which we denote by $B E R_{\text {target }}$. Delay requirements are specified at the packet level. We assume that traffic streams are organized at the MAC level in blocks of bits of constant size that we call packets. The packet delay is defined as the time between the arrival of the packet in the transmission buffer and the time of its reception by the mobile or the access point. This delay is roughly equal to the packet waiting time in the service flow transmission buffer neglecting the transmission and propagation delays. The packet delay can be directly calculated in the downlink by the access point. In the uplink, this information is known by the user that computes the PDOR and regularly signals it to the access point for the $I B F S_{P D O R}$ bandwidth management. Adequately specifying the delay requirements is challenging. We believe that the meaningful constraint is a limitation of the occurrences of large delay values. By analogy with the concept of outage used in system coverage planning, we define the concept of delay outage. A service flow is in delay outage when its packets experience a delay greater than a given application specific threshold denoted Th. We define the Packet Delay Outage Ratio (PDOR) experienced by each service flow as the percentage of packets that do not meet the delay threshold $T h$ among the total number of transmitted packets:

$$
P D O R=\frac{\text { Total flow's packets not transmitted/received on time }(\text { after } t h)}{\text { Total packet transmitted/received }} .
$$

The experienced PDOR value is tracked all along the lifetime of the service flows: at each transmission of a packet of service flow, the total number of packets whose delay exceeds the delay threshold $T h$ divided by the total number of packets transmitted since the beginning of the connection is computed. Additionally, we define the Packet Delay Outage Ratio target, denoted $P D O R_{\text {target }}{ }^{3}$, as the maximum ratio of packets that may be delivered after the delay threshold. This characterizes the delay requirements of any service flow in a generic approach. Figure 3 illustrates an example cumulative distribution of the packet delay of service flow at a given time.

The objective of the IBFS $S_{M C P D O R}$ protocol is to regulate the experienced PDOR along with the lifetime of the service flow such that its value stays below the target PDOR in each cell. This ensures the satisfaction of the delay requirements at a short time scale.

\section{PERFORMANCES EVALUATION}

\section{A. Simulation setup and assumptions}

Performance evaluation results are obtained using discrete event simulations. We consider Orthogonal Frequency Division Multiplexing (OFDM) transmission technique that has clearly emerged for future broadband wireless multimedia networks ( $4 \mathrm{G}$ and $5 \mathrm{G}$ systems) and is already widely implemented in most recent wireless systems like $802.11 \mathrm{a} / \mathrm{g}$ or 802.16 . Traffic load and channel conditions vary independently over the time for each user in each cell. The channel gain model on each subcarrier considers free space path loss and multipath Rayleigh fading [1-4]. We assume that mobiles run videoconference applications. This demanding type of applications generates a high volume of data with high sporadicity and requires tight delay constraints which substantially complicates the task of the scheduler. We assume that each mobile has only one service flow with a traffic composed of an MPEG-4 video stream [23] and an AMR voice stream [24] with a BER target taken equal to $10^{-3}$ and a delay threshold $T h$ equal to $100 \mathrm{~ms}$. The IBFS algorithm is proposed for a generic reuse-g deployment and it will be compared to the traditional REUSE-3 model. The cell numbers in a cluster are defined following Fig. 4.

\footnotetext{
${ }^{3}$ a user can be considered as dissatisfied when its PDOR overpasses $5 \%$, so PDOR target is fixed to this value in this article.
} 


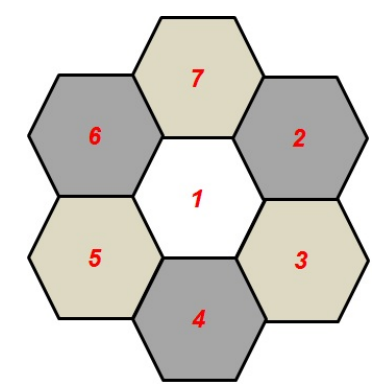

Fig. 4: Cluster illustration.

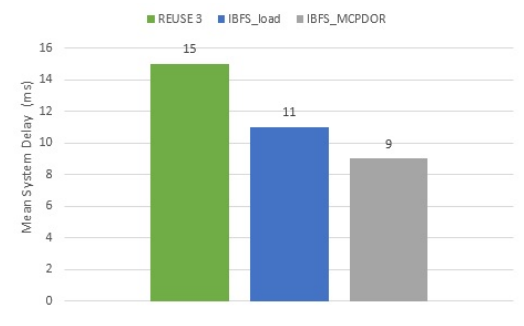

(a) Mean packet delay in the system.

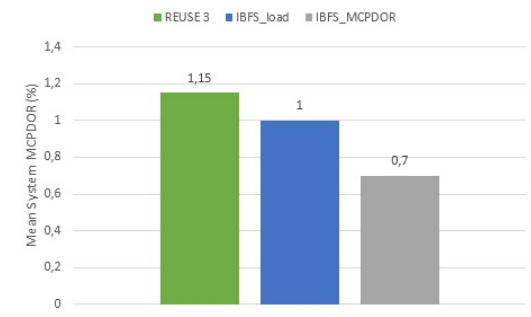

(b) Mean Packet delay Outage.

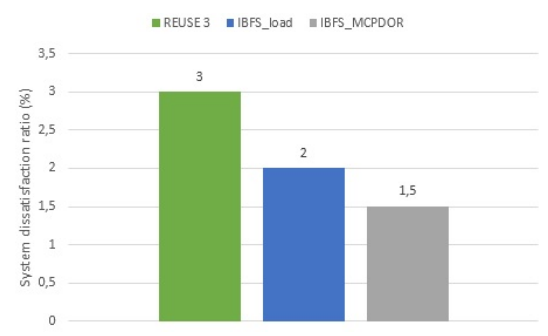

(c) Mean users dissatisfaction over the time.

Fig. 5: Simulation resuts of the first scenario

\section{B. Scenario 1: cells with Different Types of User Profiles}

In 5G wireless context, for each cell, users can have different traffic profiles. In this scenario, we assign users with high variable bit rate to the cell number 1 , while all the other cells (cell 2, 3, 4, 5, 6 and 7) have users with less variable traffic. In addition, the global traffic load of these cells is $15 \%$ higher than that of the cell 1. In this context, even with less global traffic load, cell 1 is the cell that will experience more difficulties, since high data burst can appear due to more elastic traffics that cause high delay peaks and high users dissatisfaction.

In this context, the frequency reuse- 3 model provides a MCPDOR equal to $9 \%$ in cell 1 , while the other cells have MCPDOR near to $0 \%$. This leads to high mobile dissatisfaction in cell 1 . This bad result is due to the fact that reuse-3 never helps a cell that is in difficulties so this leads to the highest global mean packet delay (Fig. 5(a)), highest MCPDOR (Fig. 5(b)) and highest user dissatisfaction (Fig. 5(c)) in the system.

$I B F S_{\text {load }}$ slightly solves this problem. It takes bandwidth from other cells when cell 1 experiences high data rate burst that overpasses other cell traffic load, in order to help it to ensure user QoE. However, sometimes, $I B F S_{\text {load }}$ can make some sub-optimal decisions. The cell that is selected to receive bandwidth (cell with the highest traffic load) may not be the cell that has the highest difficulties. It could appear that this cell has not the worst radio conditions or its delay sensitive flows are not the hardest to satisfy in the system. Based on the MCPDOR, the selection is more accurate with $I B F S_{M C P D O R}$ that outperforms $I B F S_{\text {load }}$ and widely outperforms the frequency reuse-3 model (Fig. 5(c)).

\section{Scenario 2: overloaded cells}

In this subsection we compare the performance of IBFS with that of the well-known reuse-3 technique in a scenario that illustrates a special event occurrence (like sport event, protest march, etc.). We consider exceptional gathering of users in a cell (Fig. 6). They stay in cell number 1 during a certain time, then they move to another cell (cell number 2) and come back (Fig. 7(a) and 7(b)). Cells 3, 4, 5, 6 and 7 experience normal traffic load, and have the same global cell throughput (Fig. 7(c)). 


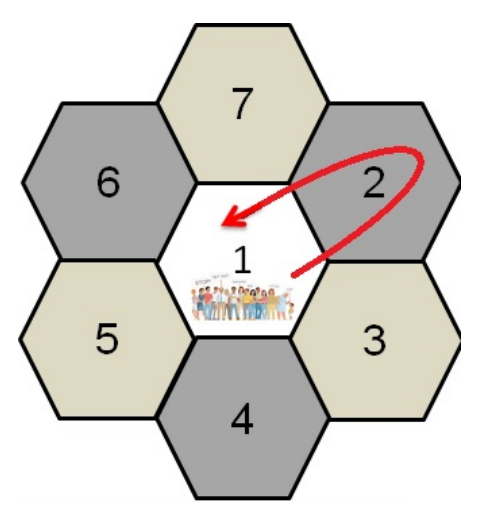

Fig. 6: performance evaluation scenario.

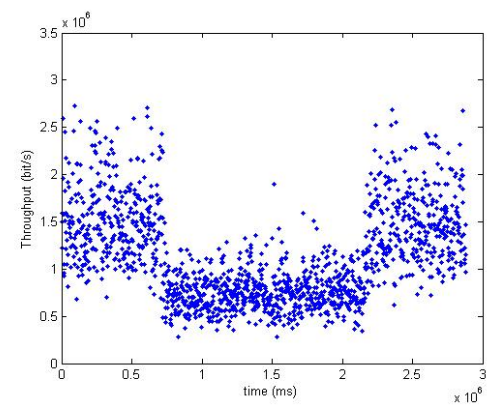

(a) Traffic load of cell 1 .

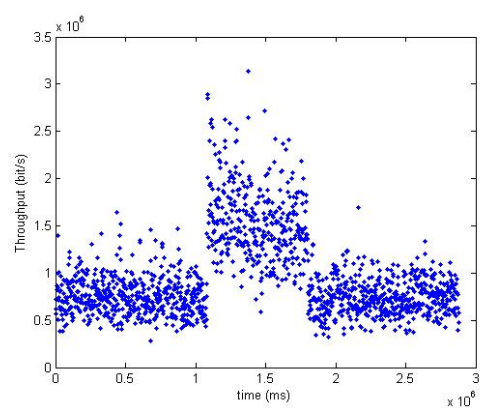

(b) Traffic load of cell 2

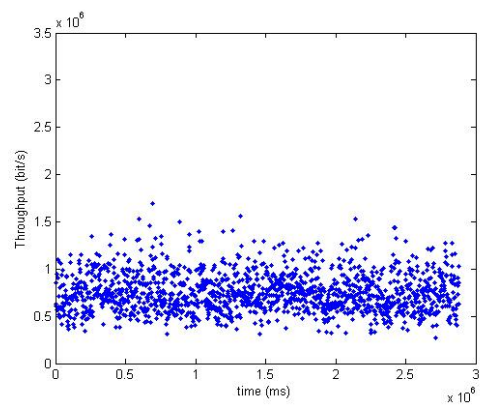

(c) Traffic load of cell 3, 4, 5, 6, 7 .

Fig. 7: System cells traffic load.

Figures 7(a), 7(b) respectively show the traffic load over time for cell 1 and 2. Figure 7(c) represents the mean traffic load of the other cells of the system that have the same mean traffic load over time. We notice that following the users moves, cell 1 is overloaded, then cell 2 and, once again cell 1.

During the first period, the cell 1 experiences high difficulties to guarantee high QoS and QoE to its users due to the high load. Figure 8 shows the average radio resources used by each solution over time. The frequency reuse- 3 model does not provide any aid to the overloaded cells. Consequently, with reuse-3, the cell 1 uses a large amount of its bandwidth (Fig. 8(a)) and sometimes has a lack of available radio resources to manage the burst of data. This induces high delay peaks as shown Fig. 9(a). On the contrary IBFS solution supports cells that experience difficulties. IBF $S_{\text {load }}$ takes bandwidth from the cells that have few data to manage in order to give radio resources to the cell that has more load. IBF $S_{M C P D O R}$ applies the same principle by selecting the cell that has the highest number of packet in delay outage in order to give it more radio resources from the other cells that experience no difficulties. According to the two solutions, more bandwidth is allocated to the cell 1 when it is overloaded, and it has consequently more capacity to absorb the data burst (Fig. 8(a)). This allows IBF $S_{l o a d}$ and IBF $S_{M C P D O R}$ to highly reduce peak of delay (Fig. 10(a), 11(a)) with a mean average packet delay in all the system equal to $23 \mathrm{~ms}$ and $22.75 \mathrm{~ms}$, respectively. However, the frequency reuse-3 technique provides a mean average packet delay equal to $67.25 \mathrm{~ms}$.

Figure 12 shows the global results of the system for the cells 1 and 2 that experience high traffic load period and for the other cells, that are represented here by the cell 4 . The frequency reuse- 3 model failed to ensure high QoE. 24\% of the packets of the cell 1 did not have their QoS constraints satisfied (Fig. 12(a)) and this results in 37\% of dissatisfaction ratio (Fig. 12(b)). This performance is slightly the same for cell 2 that experiences smaller traffic congestion over the time. On the contrary, we note that cell 4 (representative of all similar other cells that have not experienced congestion) easily succeeds to provide high QoE to its users since its available bandwidth is widely outsized. This over provisioning of 

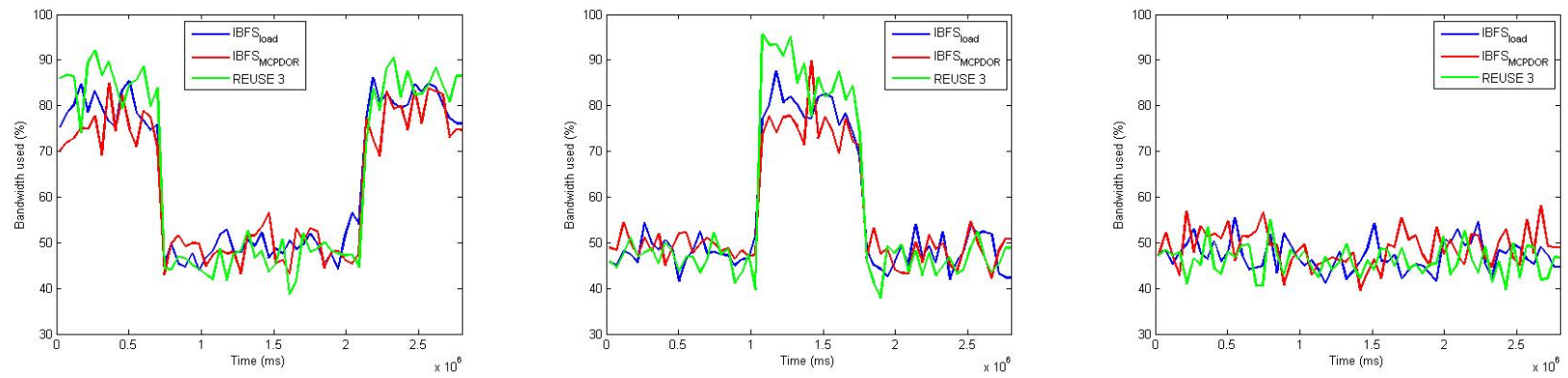

(a) Mean Bandwidth usage ratio for cell 1. (b) Mean Bandwidth usage ratio for cell 2. (c) Mean Bandwidth usage ratio for cell 3, $4,5,6,7$.

Fig. 8: REUSE3 and IBFS Bandwidth usage ratio sharing comparaison.

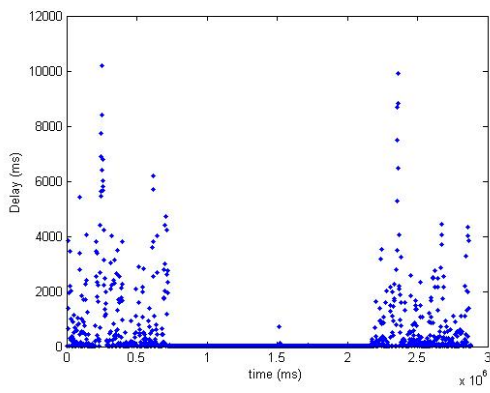

(a) Mean delay of cell 1 .

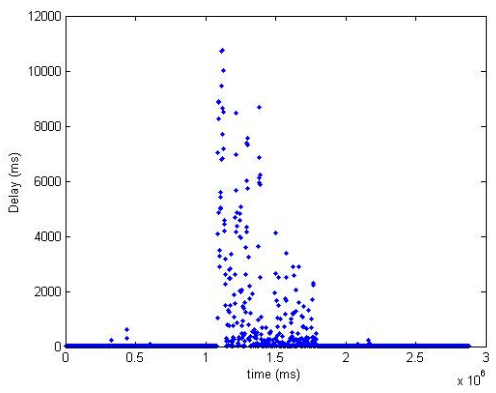

(b) Mean delay of cell 2 .

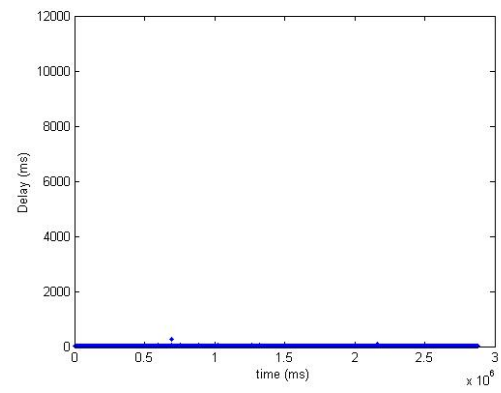

(c) Mean delay of cell 3, 4, 5, 6, 7 .

Fig. 9: System cells mean delay with REUSE3.

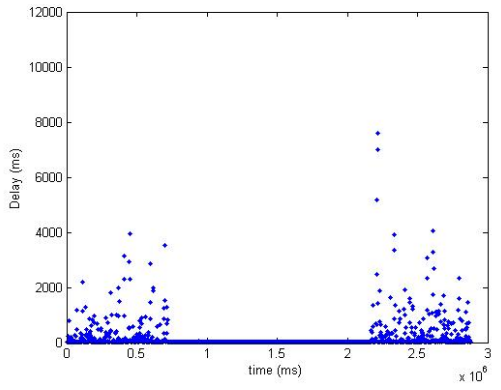

(a) Mean delay of cell 1 .

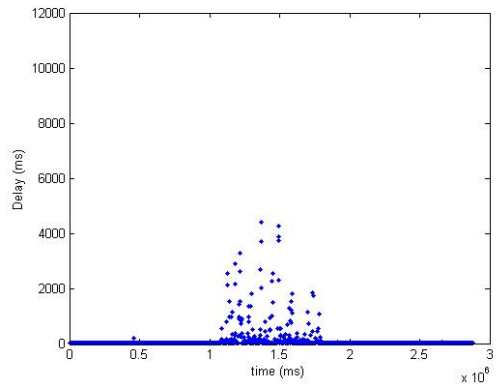

(b) Mean delay of cell 2 .

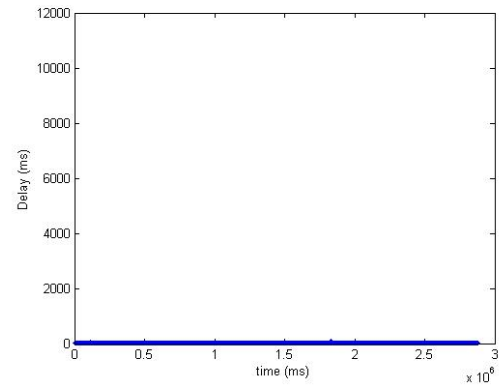

(c) Mean delay of cell 3, 4, 5, 6, 7.

Fig. 10: System cells mean delay with $I B F S_{\text {load }}$.

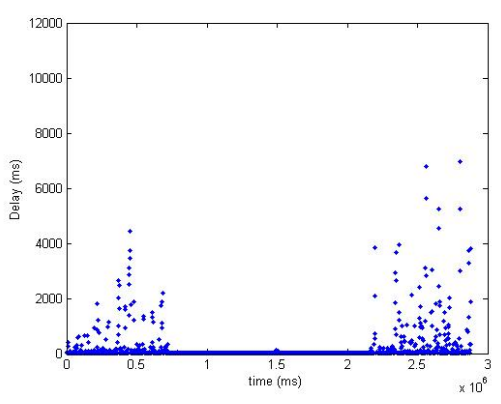

(a) Mean delay of cell 1 .

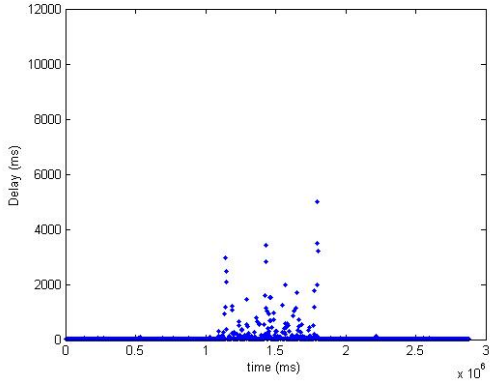

(b) Mean delay of cell 2 .

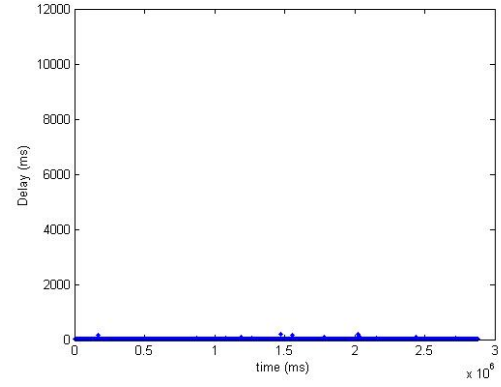

(c) Mean delay of cell 3, 4, 5, 6, 7 .

Fig. 11: System cells mean delay with $I B F S_{M C P D O R}$. 


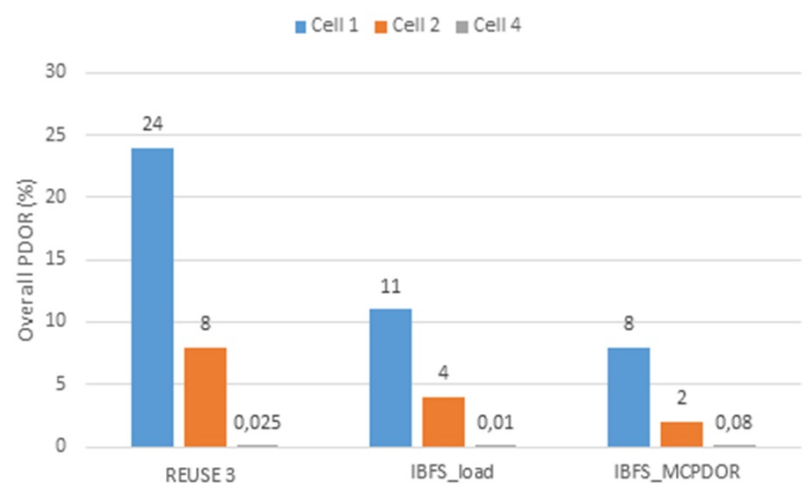

(a) Mean user PDOR in the system.

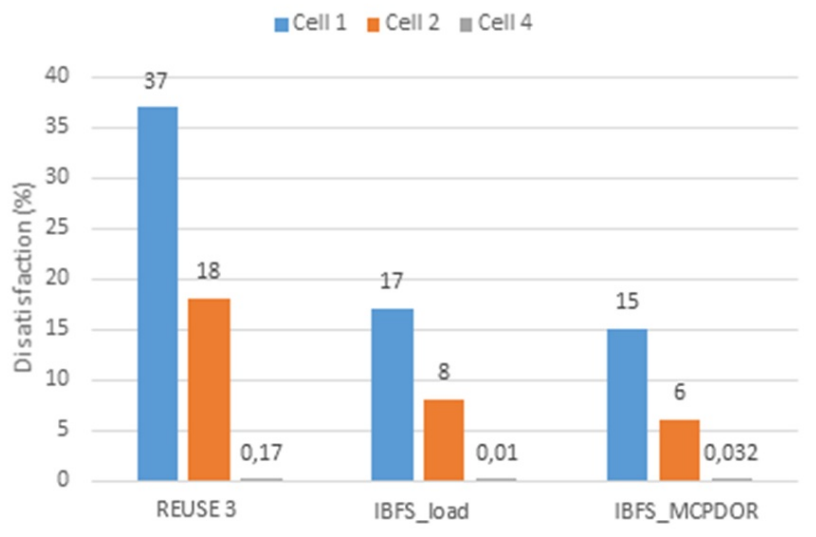

(b) Mean user dissatisfaction in the system $-P D O R_{\text {target }}=5 \%$.

Fig. 12: Analysis of the respect of QoS constraints.

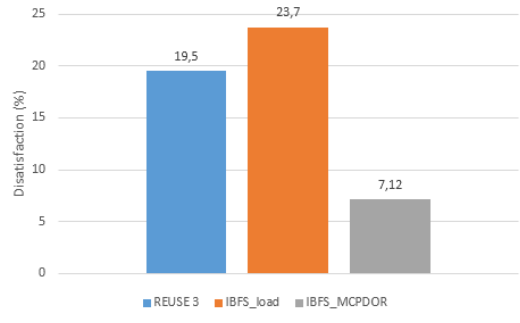

(a) Mean user dissatisfaction in cell 1

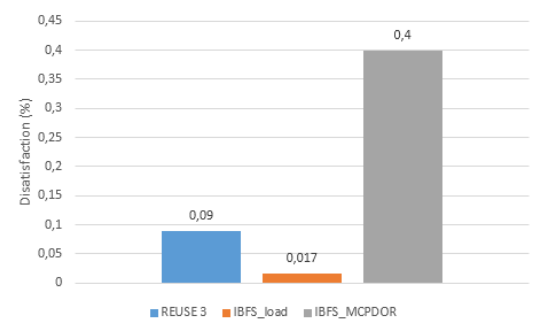

(b) Mean user dissatisfaction in cell 2, 3, 4, $5,6,7$.

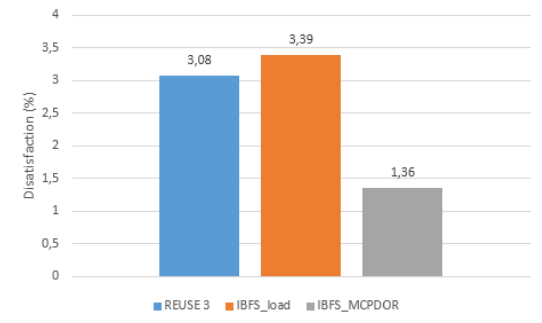

(c) Mean system's user dissatisfaction.

Fig. 13: Comparaison of users dissatisfaction for REUSE3, IBF $S_{\text {load }}$ and $I B F S_{M C P D O R}$.

useless bandwidth for the cell that does not experience difficulties to ensure QoE is a waste. If offered to overloaded cells, this bandwidth could offer high breathing space allowing to increase QoE in the system. IBFS adequately adapts bandwidth sharing between the cells that require more radio resources. This scheduler widely decreases the global amount of packets that arrive in delay outage in the period of cell overloading (Fig. 12(a)). This leads to dividing the global users dissatisfaction in cells 1 and 2 by more than 2 without penalizing the donor cells (Fig. 12(b)). In addition, these results show that the metric used to select the cell to help is crucial. They underline that IBF $S_{M C P D O R}$ outperforms $I B F S_{\text {load }}$ despite its good performance (Fig. 5, 8, 9, 10, 11, 12). This is due to the fact that high traffic load is a good indication to find the cell that might require the more assistance, but this is not sufficient enough, nor it is efficient in realistic wireless context. Delay constraints, jitter and BER must be also considered. MCPDOR verifies if these parameters are respected or not, and it is a better candidate than cell traffic load to estimate whether the scheduler succeeds or not in supporting users' traffic. The next scenario will provide complementary results that will reinforce this conclusion.

\section{Scenario 3: cells with different radio conditions}

In this scenario, we consider that all the cells have the same mean traffic load with an average of eight users per cell that are active at the same time. However, we assume that a cell (cell 1) is characterized by worst radio conditions in comparaison with other cells for a same path loss to users. For example, this can be due to a more dense/urban environment for this cell than the others which increases shadowing and multipath fading effects. Consequently, it is expected that users are more unsatisfied in cell 1 than in the other cells. 
Figure 13 shows the users dissatisfaction results: in cell 1 that provides poor radio condition (Fig. 13(a)), in all other/normal cells (Fig. 13(b)) and the average in all the system (Fig. 13(c)). Since all the cells have the same global bandwidth with Reuse-3, we notice that with this method the users of the cell 1 (that represents extremely urbanized zone) are highly penalized with $19.5 \%$ of dissatisfaction versus $0.09 \%$ in the other cells.

Despite of the good results of $I B F S_{\text {load }}$ in previous scenarii, it is interesting to observe that, in this scenario, IBF $S_{\text {load }}$ is not efficient. Since this algorithm helps the cell that has the highest traffic load and since all the cells have globally the same traffic load, it allocates the same priority and the same mean amount of bandwidth to each cell. Consequently, IBF $S_{\text {load }}$ fails to help the cell 1 to manage its users. This is not due to the fact that the principle of IBFS is inefficient in this context but just that the metric chosen for selecting the cell to help is not adequate.

Indeed, on the contrary, IBF $S_{M C P D O R}$ succeeds to help users of cell 1. Due to bad radio conditions, their PDOR quickly increases and the MCPDOR of the cell 1 surpasses the MCPDOR of normal cells. Thanks to MCPDOR, IBF $S_{M C P D O R}$ adequately detects that the cell 1 needs more bandwidth than the other cells even if the traffic load is globally the same and efficiently shares the radio resources between the cells. The result is a dissatisfaction ratio equal to $7.12 \%$ for the users of cell 1 (dissatisfaction divided by 2.74 compared to the frequency Reuse- 3 model), while the dissatisfaction of users of the other cells stays bellow $1 \%$ (results are $0.4 \%$ which can be neglected). Focusing on the global results in Fig. 13, the global user dissatisfaction is highly reduced.

\section{CONCLUSION}

Many works in intra-cellular scheduling have been done in order to increase system throughput, QoS and QoE. However, in 5G networks, many events can lead to a cell overloaded scenarii where all the current solutions fail to guarantee high user satisfaction. This article proposed a new inter-cellular MAC scheduling scheme in order to enhance the Quality of Experience in $4 \mathrm{G}$ and $5 \mathrm{G}$ wireless networks. It dynamically selects the cells that experience the highest difficulties in order to give them more radio resources from the cells that do not need them. Our solution is proposed in two versions. It helps the cell that has the highest traffic load, and the obtained results widely outperform traditional solutions. However, we are convinced that traffic load is not the most efficient criteria in order to select the cell that will receive more frequency resources. Consequently, a second contribution of this article is to take into consideration a new parameter able to always make the best decision. Simulation results and performance evaluations confirm this choice, and system performance is further improved. In future works, we will search for the optimal value of minimal bandwidth to guarantee to each cell. We will also search for the best tradeoff about the amount of bandwidth to take to the donor cell in order to optimize the beneficiary cell help, without decreasing the donor cell's QoE.

\section{REFERENCES}

[1] J. G. Proakis, Digital Communications. 3rd ed. New York: McGraw-Hill, 1995.

[2] R. Steele and L. Hanzo, Mobile Communications. IEEE Computer Society Press, 2000.

[3] A. Goldsmith, Wireless Communications. Cambridge University Press, 2005.

[4] J. D. Parsons, The Mobile Radio Propagation Channel. Wiley, 1992.

[5] L. Hanzo, J. Akhtman, L. Wang, and M. Jiang, MIMO-OFDM for LTE, WiFi and WIMAX: Coherent Versus non-Coherent and Cooperative Turbo-Transceivers, Wiley, Ed. Chichester, UK: Wiley-IEEE Press, October 2010.

[6] E. Dahlman, S. Parkvall, and J. Skold, 4G LTE and LTE-Advanced for Mobile Broadband, 1st ed., Elsevier, Ed. Oxford: Elsevier, 2011.

[7] NGMN Alliance, "5G White Paper," White Paper, Fujitsu, February 2015.

[8] A. D. Domenica and E. C. Strinati, "A Radio Resource Management Scheduling Algorithm for Self-Organizing Femtocells," in Proc. IEEE $21^{\text {st }}$ Int. Symp. Personal, Indoor, and Mobile Radio Communications, Istanbul, September 2010, pp. 191-196.

[9] V. Chandrasekhar and J. Andrews, "Spectrum Allocation in Tiered Cellular Networks," IEEE Trans. Wireless Commun., vol. 57, no. 10, pp. 3059-3068, October 2009.

[10] V. Ramachandran, V. Kamble, and S. Kalyanasundaram, "Frequency Selective OFDMA Scheduler with Limited Feedback," in IEEE Wireless Commun. Netw. Conf., Las Vegas, April 2008, pp. 1604-1609. 
[11] S. Yoon and J. Cho, "Interference Mitigation in Heterogeneous Cellular Networks of Macro and Femto Cells," in Proc. Int. Conf. ICTC, Seoul, September 2011, pp. 177-181.

[12] R. Knopp and P. Humblet, "Information capacity and power control in single-cell multiuser communications," in Proc. IEEE Int. Conf. on Communications (ICC), vol. 1, June 1995, pp. 331 - 335.

[13] C. Y. Wong and R. S. Cheng, "Multiuser OFDM with adaptive subcarrier, bit, and power allocation," IEEE J. Sel. Areas Commun., 1999.

[14] X. Wang and W. Xiang, "An OFDM-TDMA/SA MAC protocol with QoS constraints for broadband wireless LANs," ACM/Springer Wireless Networks, vol. 12, no. 2, pp. 159 - 170, 2006.

[15] METIS D6.6, "Final Report on the METIS 5G System Concept and Technology Roadmap," ICT-317669 METIS Deliverable 6.6, Version 1, April 2015.

[16] G. Fodor, C. Koutsimanis, A. Rcz, N. Reider, A. Simonsson, and W. Mller, "Intercell interference coordination in ofdma networks and in the 3gpp long term evolution system," Journal of Communications, vol. 4, pp. 445-453, 2009.

[17] A. Asheralieva and K. Mahata, "Resource Allocation for LTE-Based Cognitive Radio Network with Queue Stability and Interference Constraints," Physical Communication, vol. 14, pp. 1 - 13, 2015.

[18] J. Chen, L. Jiao, J. Wu, and X. Wang, "Fast Efficient Spectrum Allocation and Heterogeneous Network Selection Based on Modified Dynamic Evolutionary Game," Physical Communication, vol. 13, Part B, pp. 53 - 60, 2014, special Issue on Heterogeneous and Small Cell Networks.

[19] M. Yassin, M. AboulHassan, S. Lahoud, M. Ibrahim, D. Mezher, B. Cousin, and E. Sourour, "Survey of ICIC Techniques in LTE Networks under Various Mobile Environment Parameters," Accepted for publication in Springer Wireless Networks, 2015.

[20] M. Assaad, "Optimal fractional frequency reuse (ffr) in multicellular ofdma system," in Vehicular Technology Conference, 2008. VTC 2008-Fall. IEEE 68th, Calgary, Sept 2008, pp. 1-5.

[21] D. Gonzalez, M. Garcia-Lozano, S. R. Boque, and D. S. Lee, "Optimization of Soft Frequency Reuse for Irregular LTE Macrocellular Networks," IEEE Transactions on Wireless Communications, vol. 12, no. 5, pp. 2410-2423, May 2013.

[22] METIS D1.1, "Scenarios, Requirements and KPIs for 5G Mobile and Wireless System,” ICT-317669 METIS Deliverable 1.1, Version 1, April 2013.

[23] S. Baey, "Modeling MPEG4 video traffic based on a customization of the DBMAP," in Proc. Int. Symposium on Performance Evaluation of Computer and Telecommunication Systems (SPECTS), San Jose, CA, July 2004, pp. 705 - 714.

[24] P. Brady, "A model for generating on-off speech patterns in two-way conversation," Bell System Technical Journal, vol. $48,1969$. 